\title{
Phenomenological description of spin effects in electromagnetic and strong interactions of quarks
}

\author{
Alexander J. Silenko ${ }^{1,2, \star}$ and Oleg V. Teryaev ${ }^{1,3, \star \star}$ \\ ${ }^{1}$ Bogoliubov Laboratory of Theoretical Physics, Joint Institute for Nuclear Research, Dubna 141980, Russia \\ ${ }^{2}$ Research Institute for Nuclear Problems, Belarusian State University, Minsk 220030, Belarus \\ ${ }^{3}$ National Research Nuclear University "MEPhl" (Moscow Engineering Physics Institute), 115409 Moscow, \\ Russia
}

\begin{abstract}
Phenomenological description of interactions of relativistic quarks by the Dirac equation with the Cornell potential is given. The general form of the initial equation containing the vector and scalar parts of the Cornell potential is used at the arbitrary connection between these parts. The Hamiltonian in the Foldy-Wouthuysen representation is derived in the general form with allowance for the electromagnetic interactions. Unlike precedent investigations, it is relativistic and exact for terms of the zeroth and first powers in the Planck constant and also for such terms of the second power which describe contact interactions. General quantum mechanical equations of motion for the momentum and the spin are derived and the classical limit of the Hamiltonian and the equations of motion are found for the first time. A connection between the angular velocity of the quark spin precession and the force acting on it is determined. The energy of the spin-orbit interaction is rather high (of the order of $100 \mathrm{MeV}$ ). The terms describing the spin-orbit and contact interactions have opposite signs for the scalar and the vector parts of the Cornell potential. The evolution of the quark helicity and the spin-spin interaction of the quarks are also calculated.
\end{abstract}

\section{Introduction}

The Cornell potential finds numerous applications in the heavy quark physics. Among these applications, we can mention collisions of heavy ions which manifest new properties of quark-gluon plasma. It is naturally to use the conventional Cornell potential for a phenomenological description of these properties in the first approximation. The Cornell potential can be included into the Dirac equation in two possible ways. It is the most natural to consider the quark interaction like an electromagnetic interaction because the quanta of these interactions, photon and gluon, have the same spin. In this case, the Cornell potential is included into the four-potential of the Dirac equation. Another possibility is an inclusion of the Cornell potential into the scalar potential [1]. One often realizes the both possibilities and introduces the Cornell potential in the two (vector and scalar) forms [2-6]. Certainly, the vector Cornell potential is a four-vector. The use of traditional quantum-mechanical approaches allows one

\footnotetext{
$\star^{\star}$ e-mail: alsilenko@mail.ru

${ }^{\star}$ e-mail: teryaev@theor.jinr.ru
} 
to solve the resulting equation only in some specific case, when the parameters of the scalar and vector potentials are very specific [3-6].

In the present work, we use the both scalar and vector Cornell potentials and solve the more general problem of a strongly interacting Dirac particle (quark). Unlike other investigations [1-6], we use the approach based on the relativistic Foldy-Wouthuysen (FW) transformation and find a general solution of the problem for arbitrary parameters of the scalar and vector potentials. A possibility to restrict ourselves to a consideration of local interactions (because of short-range nuclear forces) allows us to realize advantages of this approach. Developed methods of the relativistic FW transformation [79] (see also [10-12] and references therein) ensure fulfilling this transformation in arbitrarily strong external fields. While this approach has not been used for strong interactions, it has proven itself as a powerful tool for a description of electromagnetic [11, 13, 14], gravitational [15-21], and weak $[22,23]$ interactions of single particles.

We use the system of units $\hbar=1, c=1$. Nevertheless, we include the Planck constant into several formulae.

\section{Cornell potential and its inclusion into the Dirac equation}

The standard approach in phenomenological description of interaction of quarks consists in the use of the Cornell potential having the form

$$
\mathfrak{B}(r)=-\frac{a(r)}{r}+b r
$$

In the first approximation, we can suppose that $a$ and $b$ are constant. In this case, $a \approx 100 \mathrm{MeV} \cdot \mathrm{fm}, b \approx$ $400 \mathrm{MeV} \cdot \mathrm{fm}^{-1}$. However, the second term in the Cornell potential is proportional to $r^{2}$ at condition that $r$ is small [24].

For a phenomenological description of interaction of quarks, the Cornell potential should be included into the Dirac equation. This can be made in different ways. There are some similarities between the strong and electromagnetic interactions of quarks. Therefore, an inclusion of the potential (1) into the four-vector potential $\mathcal{A}^{\mu}=(\mathbb{F}, \mathcal{A})$ similarly to the Coulomb interaction is the most natural. In this case, $\mathbb{F}$ and $\mathcal{A}$ are the scalar and vector potentials of the source (quark). Their dependence on the velocity of the field source $\boldsymbol{V}$ is defined by the Lorentz transformations:

$$
\mathbb{F}=\gamma \mathbb{F}_{0}, \quad \mathcal{A}=\gamma \mathbb{F}_{0} \frac{V}{c},
$$

where $\gamma=\left(1-V^{2} / c^{2}\right)^{-1 / 2}$, and $\mathbb{F}_{0}$ is the potential of the source at rest. If the scalar Cornell potential is not used, $\mathbb{F}_{0}=\mathfrak{B}(r)$.

Alternatively, the Cornell potential can be included into the Dirac equation as a scalar potential. In this case, the Cornell potential is multiplied by the matrix $\beta$, and its inclusion means the effective modification of the quark mass. The scalar and vector Cornell potentials are additive in the static limit. Therefore, the general Dirac Hamiltonian takes the form

$$
\mathcal{H}=\beta\left[m c^{2}+C_{0} \mathfrak{B}(r)\right]+e \Phi+\mathbb{F}+\boldsymbol{\alpha} \cdot \boldsymbol{\pi}, \quad \boldsymbol{\pi}=\boldsymbol{p}-e \boldsymbol{A}-\mathcal{A},
$$

where $\boldsymbol{p}=-i \hbar \nabla$ is the momentum operator. The electromagnetic interaction is characterized by the scalar $(\Phi)$ and vector $(\boldsymbol{A})$ potentials. The potentials of the strong interaction are defined by (2) and (3) where

$$
\mathbb{F}_{0}=C_{1} \mathfrak{B}(r), \quad C_{0}+C_{1}=1 .
$$


One mostly incorporated both the scalar and vector potentials into the Dirac equation [3-6]. The case of specific relations between the two incorporated potentials was the only case investigated in these works. This equality does not have any physical substantiation but it allows one to obtain exact solutions [3-6]. In fact, the scalar and vector Cornell potentials present two different independent approaches to a description of the strong interaction. In [1], the vector potential has not been used. In this work, the FW transformation was performed only for a nonrelativistic Dirac particle in the weak field approximation. In the present work, the much more general problem of a high-precision description of a relativistic quark in an arbitrarily strong field is solved thanks to the use of a more universal method of the FW transformation. This allows us to calculate the effect of the strong spinorbit interaction of relativistic quarks.

\section{Phenomenological description of strong interaction with the vector Cornell potential}

When the vector Cornell potential is used and the electromagnetic interaction of quarks is taken into account, the Dirac Hamiltonian takes the form

$$
\left(i \hbar \gamma^{\mu} D_{\mu}-m c^{2}+\frac{\mu^{\prime}}{2} \sigma^{\mu v} F_{\mu \nu}+\frac{m^{\prime}}{2} \sigma^{\mu v} \mathcal{G}_{\mu \nu}\right) \Psi=0, \quad D_{\mu}=\partial_{\mu}+i e A_{\mu}+i \mathcal{A}_{\mu} .
$$

Here $\mu^{\prime}$ and $\mathrm{m}^{\prime}$ are the anomalous magnetic and chromomagnetic moments, $F_{\mu \nu}=\partial_{\mu} A_{\nu}-\partial_{\nu} A_{\mu}$ and $\mathcal{G}_{\mu \nu}=\partial_{\mu} \mathcal{A}_{\nu}-\partial_{\nu} \mathcal{A}_{\mu}$ are the tensors of the electromagnetic and chromoelectromagnetic fields and $D_{\mu}$ is the covariant derivative. This equation generalizes the Dirac-Pauli one (see [13] and references therein). The strong interaction is here similar to the electromagnetic one.

In this case, the Hamiltonian in the Dirac representation takes the form

$$
\mathcal{H}=\beta m c^{2}+e \Phi+\mathbb{F}+\boldsymbol{\alpha} \cdot \boldsymbol{\pi}-\boldsymbol{\Pi} \cdot \boldsymbol{M}+i \boldsymbol{\gamma} \cdot \boldsymbol{P}
$$

where

$$
\begin{gathered}
\mathcal{M}=\mu^{\prime} \boldsymbol{B}+\mathfrak{m}^{\prime} \mathcal{R}, \quad \mathcal{P}=\mu^{\prime} \boldsymbol{E}+\mathfrak{m}^{\prime} \boldsymbol{Q}, \\
\boldsymbol{Q}=-\nabla \mathbb{F}-\frac{\partial \mathcal{A}}{\partial t}, \quad \mathcal{R}=\nabla \times \mathcal{A} .
\end{gathered}
$$

Here the chromoelectric and chromomagnetic fields, $\boldsymbol{Q}$ and $\mathcal{R}$, are introduced.

Now we fulfill the transformation to the FW representation [25] with the method developed in $[8,9]$. This representation holds a special place in quantum mechanics due to some unique properties. In this representation, quantum mechanical operators for relativistic particles in an external field have the same form as in the nonrelativistic quantum theory. In particular, the position operator [26] and momentum operator are equal to $\boldsymbol{r}$ and $\boldsymbol{p}=-i \hbar \nabla$, respectively, and the polarization operator for spin-1/2 particles is expressed by the Dirac matrix $\Pi$. In other representations, these operators are given by much more cumbersome formulae (see [7, 25]). The relations between the operators in the FW representation are analogous to the relations between the corresponding classical quantities. The simple form of operators corresponding to classical observables is a great advantage of this representation. The above-mentioned properties of the FW representation allow one to use it successfully for passing to the semiclassical approximation and to the classical limit of relativistic quantum mechanics $[25,27]$. We note that the Hamiltonian and all other operators are diagonal in two spinors (block-diagonal) in this representation.

When the FW representation is used, the passage to the classical limit is usually accomplished by simply replacing the operators in expressions for the Hamiltonian and in operator equations of motion with the corresponding classical quantities. The possibility of such a replacement, explicitly 
or implicitly used in practically all works devoted to the relativistic FW transformation, was rigorously proven in [10]. This possibility radically simplifies an interpretation of the basic quantum mechanical equations, especially in the relativistic case.

Initial Hamiltonian (6) can be presented in the following general form:

$$
\mathcal{H}=\beta m+\mathcal{E}+O, \quad \beta \mathcal{E}=\mathcal{E} \beta, \quad \beta O=-O \beta,
$$

where $\mathcal{E}$ and $O$ are even and odd (diagonal and off-diagonal in two spinors) operators, respectively.

The Hamiltonian transformed to the FW representation is exact for terms proportional to the zeroth and first powers of the Planck constant and also for terms describing contact interactions and proportional to $\hbar^{2}$. It is given by $[8,11]$

$$
\begin{gathered}
\mathcal{H}_{F W}=\beta \epsilon+\mathcal{E}-\frac{1}{8}\left\{\frac{1}{\epsilon(\epsilon+m)},[O,[O, \mathcal{Z}]]\right\}, \\
\mathcal{Z}=\mathcal{E}-i \frac{\partial}{\partial t}, \quad \epsilon=\sqrt{m^{2}+O^{2}}
\end{gathered}
$$

In the considered case, this Hamiltonian has the form

$$
\begin{gathered}
\mathcal{H}_{F W}=\beta \epsilon^{\prime}+e \Phi+\mathbb{F}+\frac{\hbar}{8 m^{2}}\left\{\frac{1}{\gamma(\gamma+1)},(\boldsymbol{\Sigma} \cdot[\boldsymbol{\pi} \times(e \boldsymbol{E}+\boldsymbol{Q})\right. \\
-(e \boldsymbol{E}+\boldsymbol{Q}) \times \boldsymbol{\pi}]-\hbar \nabla \cdot(e \boldsymbol{E}+\boldsymbol{Q}))\} \\
+\frac{\hbar}{8 m^{2}}\left\{\frac{1}{\gamma},\left(\boldsymbol { \Sigma } \cdot \left[\boldsymbol{\pi} \times\left(e G \boldsymbol{E}+G_{c} \boldsymbol{Q}\right)\right.\right.\right. \\
\left.\left.\left.-\left(e G \boldsymbol{E}+G_{c} \boldsymbol{Q}\right) \times \boldsymbol{\pi}\right]-\hbar \nabla \cdot\left(e G \boldsymbol{E}+G_{c} \boldsymbol{Q}\right)\right)\right\} \\
-\frac{\hbar}{4 m}\left\{\frac{1}{\gamma},(\boldsymbol{\Pi} \cdot[e \boldsymbol{B}+\mathcal{R}])\right\}-\frac{\hbar}{2 m}\left(\boldsymbol{\Pi} \cdot\left[e G \boldsymbol{B}+G_{c} \mathcal{R}\right]\right) \\
+\beta \frac{\hbar}{8 m^{3}}\left\{\frac{1}{\gamma(\gamma+1)},\left[\left(\left[e G \boldsymbol{B}+G_{c} \mathcal{R}\right] \cdot \boldsymbol{\pi}\right)(\boldsymbol{\Sigma} \cdot \boldsymbol{\pi})+(\boldsymbol{\Sigma} \cdot \boldsymbol{\pi})(\boldsymbol{\pi} \cdot[e G \boldsymbol{B}\right.\right. \\
\left.\left.\left.\left.+G_{c} \mathcal{R}\right]\right)+2 \pi \hbar\left(\boldsymbol{\pi} \cdot\left[e G \boldsymbol{j}+G_{c} \boldsymbol{I}\right]+\left[e G \boldsymbol{j}+G_{c} \mathcal{I}\right] \cdot \boldsymbol{\pi}\right)\right]\right\},
\end{gathered}
$$

where $\gamma=\epsilon^{\prime} / m=\sqrt{m^{2}+\pi^{2}} / m$ is the Lorentz factor, $G=(g-2) / 2=2 m c \mu^{\prime} /(e \hbar), G_{c}=\left(g_{c}-\right.$ 2) $/ 2=2 \mathrm{mcm}^{\prime} / \hbar, g_{c}$ is the chromomagnetic $g$ factor similar to the electromagnetic one, $\boldsymbol{j}=[\boldsymbol{\nabla} \times \boldsymbol{B}-$ $\partial \boldsymbol{E} /(\partial t)] /(4 \pi)$, and $\boldsymbol{I}=[\boldsymbol{\nabla} \times \mathcal{R}-\partial \boldsymbol{Q} /(\partial t)] /(4 \pi)$.

In the classical limit, the operator of the angular velocity of the spin precession is given by

$$
\begin{gathered}
\boldsymbol{\Omega}=\frac{1}{m c}\left\{\frac{1}{\gamma+1} \boldsymbol{\beta} \times(e \boldsymbol{E}+\boldsymbol{Q})+\boldsymbol{\beta} \times\left(e G \boldsymbol{E}+G_{c} \boldsymbol{Q}\right)\right. \\
\left.-\frac{1}{\gamma}(e \boldsymbol{B}+\mathcal{R})-\left(e G \boldsymbol{B}+G_{c} \mathcal{R}\right)+\frac{\gamma}{\gamma+1}\left[\boldsymbol{\beta} \cdot\left(e G \boldsymbol{B}+G_{c} \mathcal{R}\right)\right] \boldsymbol{\beta}\right\} .
\end{gathered}
$$

The velocity of light $c$ is explicitly included into this equation. In the classical limit, the force acting on the quark is similar to the Lorentz force:

$$
\boldsymbol{F}=\frac{d \pi}{d t}=e \boldsymbol{E}+\boldsymbol{Q}+\boldsymbol{\beta} \times(e \boldsymbol{B}+\mathcal{R}),
$$

where $\pi$ is the kinetic momentum.

It is interesting to determine a change of the direction of particle motion in external fields. The equation of motion for the unit vector of the kinetic momentum direction, $N=\boldsymbol{\pi} /|\boldsymbol{\pi}|=\boldsymbol{v} /|\boldsymbol{v}|$, has the form

$$
\frac{d \boldsymbol{N}}{d t}=\omega \times \boldsymbol{N}, \quad \omega=-\frac{1}{\gamma m c}\left[e \boldsymbol{B}+\mathcal{R}-\frac{\boldsymbol{N} \times(e \boldsymbol{E}+\boldsymbol{Q})}{\beta}\right] .
$$


The angular velocity of spin rotation relative to the kinetic momentum direction is equal to

$$
\begin{gathered}
\boldsymbol{o}=\boldsymbol{\Omega}-\boldsymbol{\omega}=\frac{1}{m c}\left\{-\left(e G \boldsymbol{B}+G_{c} \mathcal{R}\right)+\frac{\gamma}{\gamma+1} \boldsymbol{\beta}\left(\boldsymbol{\beta} \cdot\left[e G \boldsymbol{B}+G_{c} \mathcal{R}\right]\right)\right. \\
\left.-\frac{1}{\gamma^{2}-1}[\boldsymbol{\beta} \times(e \boldsymbol{E}+\boldsymbol{Q})]+\boldsymbol{\beta} \times\left(e G \boldsymbol{E}+G_{c} \boldsymbol{Q}\right)\right\} .
\end{gathered}
$$

Evidently, the quark helicity (the spin projection onto the momentum direction) is changed in magnetic and chromomagnetic fields only when the quark has the anomalous magnetic and chromomagnetic moments, respectively. The change of the quark helicity in electric and chromoelectric fields depends on both the normal and anomalous magnetic and chromomagnetic moments, respectively.

Let us consider the interaction of two quarks. Let $\boldsymbol{V}$ be the velocity of the quark which is the source of the strong and electromagnetic fields. If we neglect the spin-spin interaction of the quarks in the first approximation, we obtain the following relations

$$
\boldsymbol{B}=\frac{\boldsymbol{V}}{c} \times \boldsymbol{E}, \quad \mathcal{R}=\frac{\mathcal{V}}{c} \times \boldsymbol{Q} .
$$

When the interacting quarks belong to different nuclei, the vectors $\boldsymbol{v}$ and $\boldsymbol{V}$ have opposite directions in the laboratory frame. At collisions of high energy nuclei, it can be often supposed that $|\boldsymbol{v}| \approx|\boldsymbol{V}| \approx c$. An opposite situation takes place when the vectors $\boldsymbol{v}$ and $\boldsymbol{V}$ are mainly co-directed. This situation can probably be realized in quark-gluon plasma due to a predominance of local interactions.

Let us use the center-of-mass system and consider the simplest (but practically very important) case of a collision of two nucleons. In this case, $\boldsymbol{V}=-\boldsymbol{v}$. Let us determine the Hamiltonian of the first quark in the field of the second one. We restrict ourselves by a phenomenological description of main effects under peripheral collisions of relativistic nuclei. To perform a more precise quantitative analysis, one needs to take into account effects of retardation and to use the Breit equation as an initial one. Nevertheless, the approach used gives us a reasonable description of the force acting on the quark and of the torque acting on its spin.

When the electromagnetic interactions are neglected, the classical limit of the Hamiltonian (10) is given by

$$
\mathcal{H}=\epsilon^{\prime}+\mathbb{F}+\frac{1}{m c}\left(\frac{1}{\gamma}+\frac{1}{\gamma+1}+2 G_{c}\right) \boldsymbol{s} \cdot(\boldsymbol{\beta} \times \boldsymbol{Q})
$$

Here $s$ is the classical spin vector. In this approximation, the angular velocity of the spin precession is equal to

$$
\mathbf{\Omega}=\frac{1}{m c}\left(\frac{1}{\gamma}+\frac{1}{\gamma+1}+2 G_{c}\right) \boldsymbol{\beta} \times \boldsymbol{Q} .
$$

We take into account that the contribution of the strong interaction into the spin precession is predominant for any energy.

It is rather convenient to express the angular velocity of the spin precession $\boldsymbol{\Omega}$ in the lab frame (more precisely, in the center-of-mass one) in terms of the force (12) in this frame. In the considered case, the force is given by

$$
\boldsymbol{F}=\left(2-\frac{1}{\gamma^{2}}\right)(e \boldsymbol{E}+\boldsymbol{Q})-\boldsymbol{\beta}(\boldsymbol{\beta} \cdot[e \boldsymbol{E}+\boldsymbol{Q}]) .
$$

When only the strong interaction is taken into account, the angular velocity of spin precession is therefore expressed as follows:

$$
\boldsymbol{\Omega}=\frac{1}{m c}\left(\frac{1}{\gamma}+\frac{1}{\gamma+1}+2 G_{c}\right) \frac{\gamma^{2}}{2 \gamma^{2}-1} \boldsymbol{\beta} \times \boldsymbol{F} .
$$


Since the force $\boldsymbol{F}$ defines the change of the momentum, it may be in principle extracted from experimental data. Thus, (19) is convenient for an analysis of experimental effects.

The sign of the projection of $\boldsymbol{\Omega}$ onto the normal to the scattering plane depends on the sign of $G_{c}$. If $G_{c}<0$ (see $[28,29]$ ) and the Lorentz factor is large enough, the vectors $\boldsymbol{\Omega}$ and $\boldsymbol{\beta} \times \boldsymbol{F}$ are oppositely directed.

Equation (19) defines the rather large angular velocity of the spin precession which corresponds to the rather high energy of the spin-orbit interaction (of the order of $100 \mathrm{MeV}$ ). The angular velocity of spin precession is of the same order of magnitude as the vorticity calculated in [30].

It is important to give a semiclassical description of evolution of the quark helicity. When we consider the case of a collision of two nucleons and neglect the spin-spin interaction of the quarks, we can use the relations (15) and $\mathcal{V}=-\boldsymbol{v}$. In this case, (14) takes the form

$$
\boldsymbol{o}=\frac{1}{m c}\left[e\left(2 G-\frac{1}{\gamma^{2}-1}\right) \boldsymbol{\beta} \times \boldsymbol{E}+\left(2 G_{c}-\frac{1}{\gamma^{2}-1}\right) \boldsymbol{\beta} \times \boldsymbol{Q}\right] .
$$

Taking into account the spin-spin interaction can also be necessary in some cases. In electrodynamics, the field of the immobile magnetic dipole $\tilde{\mu}$ is given by

$$
\boldsymbol{B}=\frac{3 \boldsymbol{n}(\tilde{\boldsymbol{\mu}} \cdot \boldsymbol{n})-\tilde{\boldsymbol{\mu}}}{r^{3}} .
$$

The field of a moving magnetic dipole is defined by the Lorentz transformations.

The Hamiltonian of interaction of the immobile magnetic dipole $\boldsymbol{\mu}$ with the magnetic field $\boldsymbol{B}$ reads

$$
\mathcal{H}_{\text {int }}=-\boldsymbol{\mu} \cdot \boldsymbol{B}=-\frac{3(\boldsymbol{\mu} \cdot \boldsymbol{n})(\tilde{\boldsymbol{\mu}} \cdot \boldsymbol{n})-(\boldsymbol{\mu} \cdot \tilde{\boldsymbol{\mu}})}{r^{3}} .
$$

Since the magnetic dipole moment is proportional to the spin $[\mu=e g s /(2 m c)]$, the Hamiltonian of interaction (21) corresponds to the Thomas-Bargmann-Michel-Telegdi equation [31, 32] (see also $[13,33])$ in the general case. This equation describes the spin motion in electromagnetic fields:

$$
\begin{gathered}
\mathcal{H}_{\text {int }}=-\boldsymbol{\Omega} \cdot \boldsymbol{s}, \quad \boldsymbol{\Omega}=-\frac{e}{m c}\left[\left(G+\frac{1}{\gamma}\right) \boldsymbol{B}\right. \\
\left.-\frac{\gamma G}{\gamma+1}(\boldsymbol{\beta} \cdot \boldsymbol{B}) \boldsymbol{\beta}-\left(G+\frac{1}{\gamma+1}\right) \boldsymbol{\beta} \times \boldsymbol{E}\right],
\end{gathered}
$$

where $\boldsymbol{B}$ and $\boldsymbol{E}$ are the fields created by the magnetic dipole $\tilde{\boldsymbol{\mu}}$. The spin-spin interaction of quarks conditioned by the vector Cornell potential has similar properties.

Thus, the strong interaction of quarks leads to the spin-orbit interaction which results in a spin rotation and in a preferable orientation of the spin. The latter effect is similar to a spin polarization in targets placed in a magnetic field. In the case of strange quarks, this effect can be an additional explanation of hyperon polarization [30]. We can also state the existence of the spin-spin interaction and the possibility of a significant change of the quark helicity.

\section{Dirac equation with the scalar and vector Cornell potentials}

Let us consider the general case when the Dirac equation contains both the scalar and vector Cornell potentials. This equation was investigated in the recent works [2-6]. In these works, an analysis has been made only for specific values of $C_{0}$ and $C_{1}$. These values have been chosen by reason of symmetry. However, it is difficult to substantiate the corresponding symmetry of the Dirac equation by any physical arguments. The method of the FW transformation used in the present work allows us to derive the relativistic Hamiltonian for arbitrary $C_{0}$ and $C_{1}$. Certainly, these coefficients should satisfy the relation (4). 
With the use of (8) and (9), we obtain the following result:

$$
\begin{gathered}
\mathcal{H}_{F W}=\beta \epsilon^{\prime}+e \Phi+\mathbb{F}+\frac{\hbar}{8 m^{2}}\left\{\frac{1}{\gamma(\gamma+1)},(\boldsymbol{\Sigma} \cdot[\boldsymbol{\pi} \times(e \boldsymbol{E}+\boldsymbol{Q})\right. \\
-(e \boldsymbol{E}+\boldsymbol{Q}) \times \boldsymbol{\pi}]-\boldsymbol{\Pi} \cdot(\boldsymbol{\pi} \times \boldsymbol{E}-\boldsymbol{E} \times \boldsymbol{\pi})-\hbar \nabla \cdot(e \boldsymbol{E}+\boldsymbol{Q}) \\
+\beta \hbar \nabla \cdot \boldsymbol{E})\}+\frac{\hbar}{8 m^{2}}\left\{\frac{1}{\gamma},\left(\boldsymbol { \Sigma } \cdot \left[\boldsymbol{\pi} \times\left(e G \boldsymbol{E}+G_{c} \boldsymbol{Q}\right)\right.\right.\right. \\
\left.\left.\left.-\left(e G \boldsymbol{E}+G_{c} \boldsymbol{Q}\right) \times \boldsymbol{\pi}\right]-\hbar \nabla \cdot\left(e G \boldsymbol{E}+G_{c} \boldsymbol{Q}\right)\right)\right\} \\
-\frac{\hbar}{4 m}\left\{\frac{1}{\gamma},(\boldsymbol{\Pi} \cdot[e \boldsymbol{B}+\mathcal{R}])\right\}-\frac{\hbar}{2 m}\left(\boldsymbol{\Pi} \cdot\left[e G \boldsymbol{B}+G_{c} \mathcal{R}\right]\right) \\
+\beta \frac{\hbar}{8 m^{3}}\left\{\frac{1}{\gamma(\gamma+1)},\left[\left(\left[e G \boldsymbol{B}+G_{c} \mathcal{R}\right] \cdot \boldsymbol{\pi}\right)(\boldsymbol{\Sigma} \cdot \boldsymbol{\pi})\right.\right. \\
\quad+(\boldsymbol{\Sigma} \cdot \boldsymbol{\pi})\left(\boldsymbol{\pi} \cdot\left[e G \boldsymbol{B}+G_{c} \mathcal{R}\right]\right) \\
\left.\left.+2 \pi \hbar\left(\boldsymbol{\pi} \cdot\left[e G \boldsymbol{j}+G_{c} \boldsymbol{I}\right]+\left[e G \boldsymbol{j}+G_{c} \boldsymbol{I}\right] \cdot \boldsymbol{\pi}\right)\right]\right\} .
\end{gathered}
$$

The analysis of the Hamiltonian (23) brings the following conclusions. The terms describing the spin-orbit and contact interactions have opposite signs for the scalar and vector Cornell potentials. The energy of the spin-orbit interaction is rather high and is of the order of $100 \mathrm{MeV}$. We can also mention that the transition to the classical limit of (23) reduces to the replacement of the operators in this equation with the corresponding classical quantities. In particular, the spin operators $\hbar \boldsymbol{\Sigma}$ and $\hbar \boldsymbol{\Pi}$ are substituted by the doubled classical spin vector, $2 s$.

\section{Summary}

We have considered the strong and electromagnetic interactions of Dirac particles (quarks). We have used the scalar and vector Cornell potentials for a phenomenological description of the strong interaction. The vector Cornell potential is similar to the four-vector potential of the electromagnetic field. Since the strong interaction is much closer to the electromagnetic interaction than to the scalar one, the use of the vector Cornell potential is more natural. Unlike previous investigations $[1,3,5,6]$, we have used the method of the relativistic FW transformation [7-9] applicable in arbitrarily strong external fields and have determined the relativistic FW Hamiltonian. In this Hamiltonian, the terms of the zeroth and first orders in the Planck constant and such terms of the order of $\hbar^{2}$ which describe contact interactions are exact. We have found the exact classical limit of this Hamiltonian. The adequate and exact classical limit of the strong interaction of quarks characterized by the Cornell potential has been determined for the first time. We also have taken into account the electromagnetic interaction which may not be negligible. Effective fields acting on the particle and spin have been found. General formulae for the angular velocity of spin precession have been derived. These formulae correspond to the high energy of the spin-orbit interaction (of the order of $100 \mathrm{MeV}$ ) for both the vector and scalar Cornell potentials. The terms describing the spin-orbit and contact interactions have opposite signs for the scalar and vector Cornell potentials. Dynamics of the quark helicity has been calculated and the spin-spin interaction of quarks has been considered. We have determined the relations between the angular velocity of spin precession and the force acting on the quark. Since this force defines the change of the momentum, it can be in principle extracted from experimental data.

\section{Acknowledgments}

This work was supported in part by the Belarusian Republican Foundation for Fundamental Research (Grant No. $\Phi 16 \mathrm{D}-004$ ) and by the Heisenberg-Landau program of the German Ministry for Science and Technology (Bundesministerium für Bildung und Forschung). 


\section{References}

[1] U.D. Jentschura, J.H. Noble, J. Phys. A: Math. Theor. 47, 045402 (2014)

[2] V.Yu. Lazur, O.K. Reity, V.V. Rubish, Phys. Rev. D 3, 076003 (2011)

[3] H. Hassanabadi, E. Maghsoodi, S. Zarrinkamar, Ann. Phys. (Berlin) 525, 944 (2013)

[4] H. Hassanabadi, E. Maghsoodi, S. Zarrinkamar, H. Rahimov, Adv. High Energy Phys. 2012, 707041 (2012)

[5] M. Hamzavi, A.A. Rajabi, Ann. Phys. (New York) 334, 316 (2013). M. Hamzavi, A.A. Rajabi, Chin. Phys. B 22, 090301 (2013). M. Hamzavi, A.A. Rajabi, Chin. Phys. C 37, 103102 (2013). M. Hamzavi, A.A. Rajabi, Few-Body Syst. 54, 2067 (2013)

[6] L.A. Trevisan, C. Mirez, F.M. Andrade, Few-Body Syst. 55, 1055 (2014)

[7] A.J. Silenko, J. Math. Phys. 44, 2952 (2003)

[8] A.J. Silenko, Phys. Rev. A 77, 012116 (2008)

[9] A.J. Silenko, Phys. Rev. A 91, 022103 (2015)

[10] A.J. Silenko, Phys. Part. Nucl. Lett. 10, 91 (2013)

[11] A.J. Silenko, Theor. Math. Phys. 176, 987 (2013)

[12] A.J. Silenko, Phys. Rev. A 91, 012111 (2015)

[13] A.J. Silenko, Theor. Math. Phys. 105, 1224 (1995)

[14] A.J. Silenko, Russ. Phys. J. 48, 788 (2005)

[15] A.J. Silenko, O.V. Teryaev, Phys. Rev. D 71, 064016 (2005)

[16] A.J. Silenko, O.V. Teryaev, Phys. Rev. D 76, 061101(R) (2007)

[17] Yu.N. Obukhov, A.J. Silenko, O.V. Teryaev, Phys. Rev. D 80, 064044 (2009)

[18] Yu.N. Obukhov, A.J. Silenko, O.V. Teryaev, Phys. Rev. D 84, 024025 (2011)

[19] Yu.N. Obukhov, A.J. Silenko, O.V. Teryaev, Phys. Rev. D 88, 084014 (2013)

[20] Yu.N. Obukhov, A.J. Silenko, O.V. Teryaev, Phys. Rev. D 90, 124068 (2014)

[21] Yu.N. Obukhov, A.J. Silenko, O.V. Teryaev, Phys. Rev. D 94, 044019 (2016)

[22] A.J. Silenko, Nuclear Instrum. Methods in Phys. Res. B 114, 259 (1996)

[23] A.J. Silenko, Theor. Math. Phys. 112, 922 (1997)

[24] Ya.Ya. Balitskii, Nucl. Phys. B 254, 166 (1983)

[25] L.L. Foldy, S.A. Wouthuysen, Phys. Rev. 78, 29 (1950)

[26] T.D. Newton, E.P. Wigner, Rev. Mod. Phys. 21, 400 (1949)

[27] J.P. Costella, B.H.J. McKellar, Am. J. Phys. 63, 1119 (1995)

[28] D. Diakonov, Prog. Part. Nucl. Phys. 51, 173 (2003)

[29] N. Kochelev, N. Korchagin, Phys. Lett. B 729, 117 (2014)

[30] M. Baznat, K. Gudima, A. Sorin, O. Teryaev, Phys. Rev. C 88, 061901 (2013)

[31] L.H. Thomas, Nature (London) 117, 514 (1926). L.H. Thomas, Philos. Mag. 3, 1 (1927)

[32] V. Bargmann, L. Michel and V.L. Telegdi, Phys. Rev. Lett. 2, 435 (1959)

[33] A. J. Silenko, Phys. Scripta 90, 065303 (2015) 